\title{
DEVELOPMENT OF FIELD-DEPLOYABLE DIODE-LASER-BASED WATER VAPOR DIAL
}

\author{
Phong Pham Le Hoai ${ }^{1 *}$, Makoto Abo ${ }^{1}$, Tetsu Sakai ${ }^{2}$ \\ ${ }^{1}$ Graduate School of System Design, Tokyo Metropolitan University, Tokyo 191-0065, Japan, \\ *Email: phamlehoaiphong@gmail.com \\ ${ }^{2}$ Meteorological Research Institute, Tsukuba, Ibaraki 305-0052, Japan
}

\begin{abstract}
In this paper, a field-deployable diode-laser-based differential absorption lidar (DIAL) has been developed for lower-tropospheric water vapor observation in Tokyo, Japan. A photoacoustic cell is used for spectroscopy experiment around absorption peaks of $829.022 \mathrm{~nm}$ and $829.054 \mathrm{~nm}$. The water vapor density extracted from the observational data agrees with the referenced radiosonde data. Furthermore, we applied modulated pulse technique for DIAL transmitter. It enables DIAL to measure water vapor profile for both low and high altitude regions.
\end{abstract}

\section{INTRODUCTION}

Water vapor mostly exists in the planetary boundary layer, a bottom part of the troposphere. The water vapor variation is one of the major factors causing the Earth's weather phenomena, climate change, and the other atmospheric activities. Hence, a precise and high-resolution water vapor profile is necessary for the weather forecast and climate study. Lidar, an active optical remote sensing has been utilized for water vapor monitoring. There are two typical techniques of lidar including DIAL and Raman lidar.

Raman lidar technique can measure highresolution vertical profile of multiple gases simultaneously. The scattering molecule is basically identified from the frequency shift caused by the inelastic Raman scattering. It requires a high power laser pulse transmitter and a large aperture telescope to be able to receive small scattering cross section. The Raman lidar also needs periodic calibration device.

DIAL technique utilizes a pair of lasers tuned on and off the gas absorption peak, referred as online and offline laser signal. The molecular number density is basically obtained from the ratio of return online and offline signals as well as the prior knowledge of molecular absorption coefficients at online and offline wavelengths. The DIAL is able to cancel the calibration issue in Raman lidar. Furthermore, a diode laser based DIAL with the characteristics of low laser pulse energy and high pulse repetition is eye-safe, low cost, compact, and deployable.

A field-deployable diode-laser-based water vapor DIAL has been developed by the researchers at Montana State University through several generation systems. The DIAL prototype is able for continuous and autonomous operation during long-period measurement test [1]. Based on this initial work, we aim at developing a water vapor DIAL for heavy rain prediction during the summer season in urban areas of Japan.

\section{DIAL INSTRUMENT}

\subsection{Laser transmitter}

The block diagram of the laser transmitter is shown in Fig. 1. The output from a pair of distributed Bragg reflector (DBR) diode lasers are collimated using aspheric lens. The laser beam profile is reshaped by an anamorphic prism pairs (AP). Then the laser beam passes a Faraday isolator to prevent feedback and a half-wave plate $(\lambda / 2)$ to align polarization. Afterward, the laser beam is coupled in a single mode polarization maintaining fiber.

Both laser beams, then direct to a $2 \times 1$ solid-state high-speed fiber optical switch (SW) driven by a pulse generator. The seeding laser, switching between the online and offline lasers, passes through a half-wave plate and a Faraday isolator before injected to a tapered semiconductor optical amplifier (TA). The output laser beam of TA is circularly shaped by spherical and cylindrical 
lenses. Then, the laser beam is expanded for eye safety before transmitting to the air.

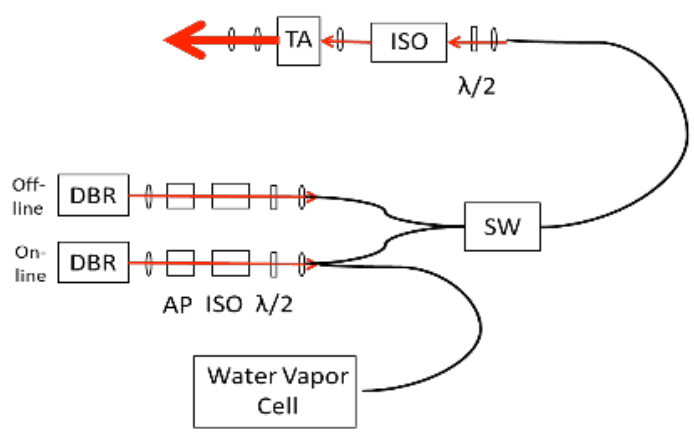

Figure 1. Block diagram of the laser transmitter.

\subsection{Absorption spectroscopy experiment}

In the scope of this work, we conducted absorption spectroscopy experiment utilizing the photoacoustic cell to measure water vapor absorption around operating wavelengths. A strong water vapor absorption peak at $829.022 \mathrm{~nm}$ and a weaker absorption peak at $829.054 \mathrm{~nm}$ used for this experiment are shown in Fig. 2.

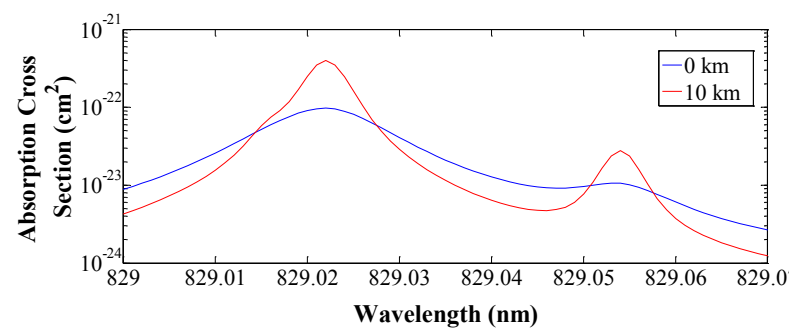

Figure 2. Voigt profile of the water vapor absorption cross section around the operational lines.

Photoacoustic spectroscopy is a sensitive technique for small absorption measurement. Fundamentally, a laser beam is directed to the absorption cell and the pressure variation in the cell is detected by a sensitive microphone, as shown in Fig. 3. The output signal of the microphone is proportional to the absorption cross section in the wavelength that the incident laser beam is tuned to. Then the output signal of microphone passes through an amplifier, a $1.5 \mathrm{kHz}-$ to- $-2.5 \mathrm{kHz}$ band pass filter, and a lock-in amplifier. Fig. 4 and Fig. 5 present the normalized absorption cross section from spectroscopy experiment using the photoacoustic cell and the referenced Voigt profiles around $829.022 \mathrm{~nm}$ $829.054 \mathrm{~nm}$, respectively. The measured absorption peaks are shifted by $1 \mathrm{pm}$ compared to the theoretical absorption peaks in both cases. The shifted wavelengths may relate to the calibration issue of the wavemeter.

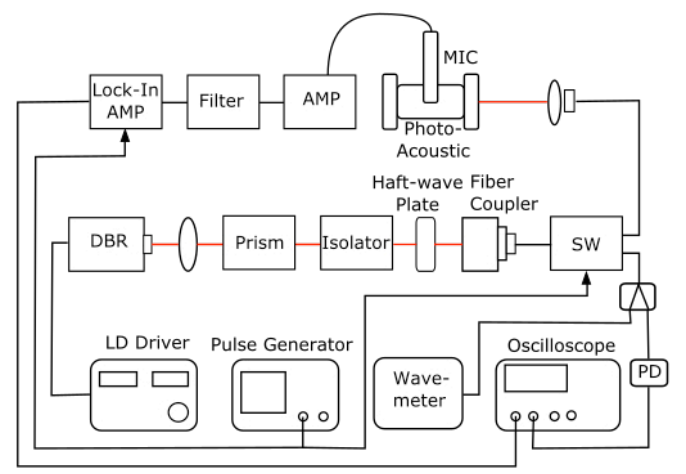

Figure 3. Schematic of spectroscopy experiment setup using the photoacoustic cell.

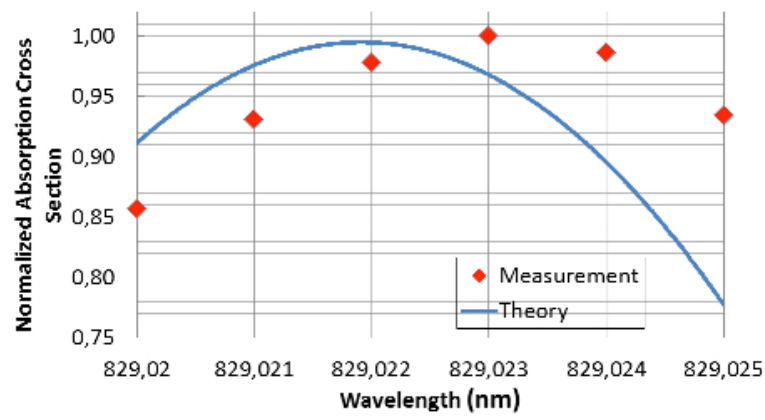

Figure 4. Normalized absorption cross section from the photoacoustic cell experiment and Voigt profile around $829.022 \mathrm{~nm}$.

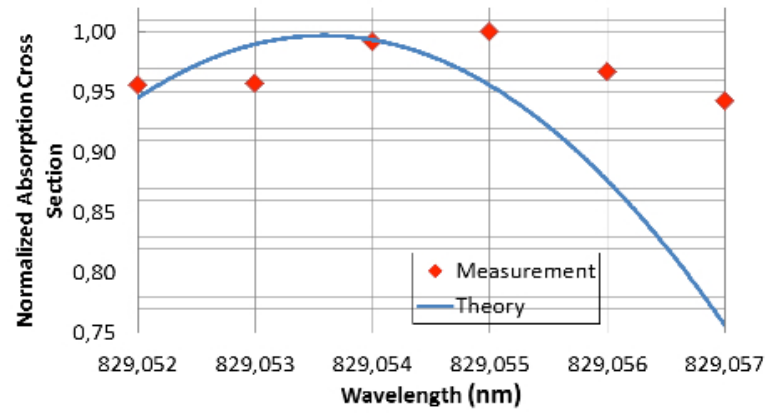

Figure 5. Normalized absorption cross section from the photoacoustic cell experiment and Voigt profile around $829.054 \mathrm{~nm}$.

\section{TEST OBSERVATION}

The DIAL receiver includes a $50 \mathrm{~cm}$ diameter telescope, a photomultiplier tube (PMT), and a lidar transient recorder. The DIAL parameters for testing observation are described in Table 1 . The photon-count numbers of the online and offline signals after subtracting background are plotted in 
Fig. 6. The water vapor density with a range resolution of $450 \mathrm{~m}$ is compared to the referenced radiosonde data, as shown in Fig. 7. As a result, the trend of lidar data coincides with the radiosonde data. However, the recent DIAL can not observe low region water vapor density due to the limit of driving pulse width.

Table 1. The $\mathrm{H}_{2} \mathrm{O}$ DIAL experimental parameters

\begin{tabular}{|l|l|}
\hline Online wavelength & $829.054 \mathrm{~nm}$ \\
\hline Offline wavelength & $829.124 \mathrm{~nm}$ \\
\hline Pulse energy & $0.24 \mu \mathrm{J}$ \\
\hline Pulse width & $3 \mu \mathrm{s}$ \\
\hline Repetition rate & $1 \mathrm{kHz}$ \\
\hline Accumulation time & 1 hour \\
\hline Optical efficiency of filter & $50 \%$ \\
\hline Quantum efficiency & $10 \%$ \\
\hline Telescope diameter & $50 \mathrm{~cm}$ \\
\hline Field of view & $3 \mathrm{mrad}$ \\
\hline
\end{tabular}

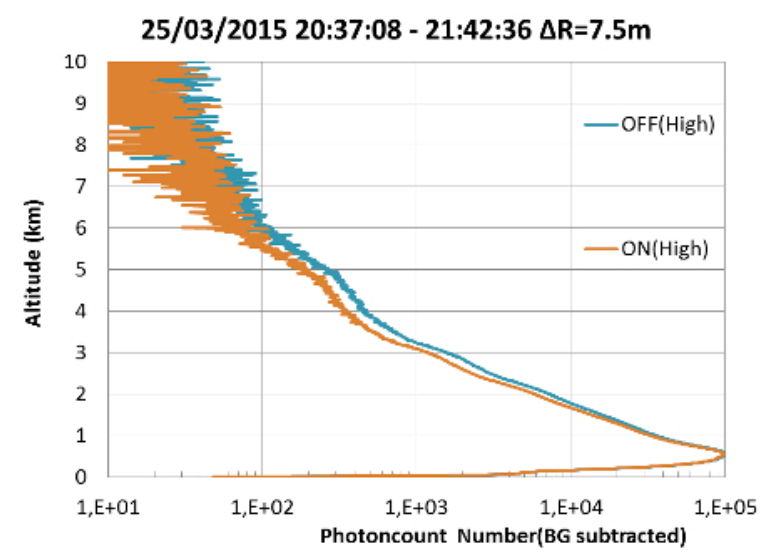

Figure 6. The photon-count number of the online and offline signals after subtracting background.

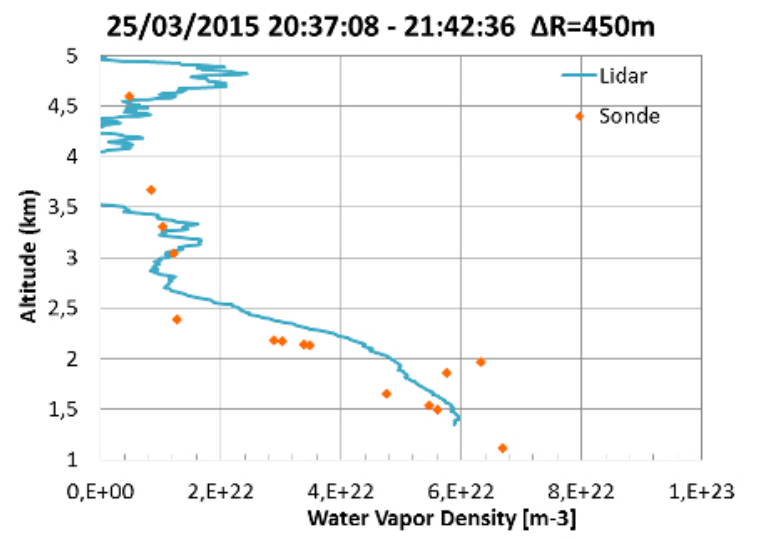

Figure 7. Lidar water vapor density with a rangeresolution of $450 \mathrm{~m}$ and the referenced radiosonde data.

\section{MODULATED PULSE TECHNIQUE}

The concept of modulated pulse technique is illustrated in Fig. 8 [2]. A single macropulse, transmitting $\mathrm{N}$ subpulses, is repeated every period of $t_{r e p}$. The width of a single macropulse is given as

$$
t_{\text {macro }}=N . t_{c}=N . t_{b}, .
$$

where subpulse width $t_{c}$ equals to bit width $t_{b}$. The waveform of a single macropulse laser is determined by

$$
x(t)=\sum_{i=0}^{N-1} a_{i} p\left(t-i t_{b}\right),
$$

where $a_{i}$, defined by pseudorandom sequence, equals 1 or 0 , and $p(t)$, the envelope of a subpulse, is described as

$$
p(t)=\left\{\begin{array}{cc}
1 & \left(0 \leq t \leq t_{b}\right) \\
0 & \text { otherwise }
\end{array} .\right.
$$

Similarly, the envelope of a macropulse is described as

$$
m(t)=\left\{\begin{array}{rr}
1 & \left(0 \leq t \leq t_{\text {macro }}\right) \\
0 & \text { otherwise }
\end{array} .\right.
$$

Consequently, the complete laser waveform is given as

$$
s(t)=\sum_{j=0}^{\infty} x(t) m\left(t-j t_{\text {rep }}\right) .
$$

At the receiver, the return signal is a convolution form of modulated laser pulse, the medium response impulse $h(\tau)$ (i.e., backscattering response), and background noise $n(t)$ (i.e., Poisson noise). The corresponding return waveform $y(t)$ of a single modulated macropulse $x(t)$ is given as

$$
y(t)=\int_{0}^{t_{\text {rep }}} x(t-\tau) h(\tau) d \tau+n(t) .
$$

Finally, the return signal $z(t)$ is demodulated by cross-correlation of $x(t)$ and $y(t)$ as

$$
z(t)=R_{y x}(\tau)=\int_{0}^{t_{r e p}} y(t) x(t-\tau) d t
$$




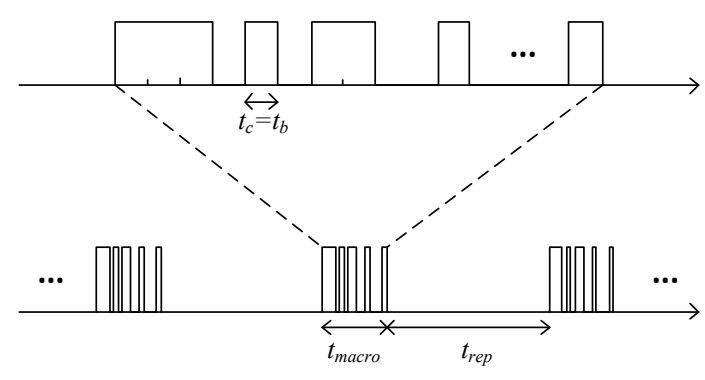

Figure 8. The concept of modulated pulse laser technique.

Theoretically, a periodic cross-correlation $z(t)$ of the continuous and periodic return signal $y(t)$ leads to a neglected sidelobe result. However, we can not keep the transmitted signal continuously in reality. By operating simultaneously a pair of online and offline wavelengths, the sidelobe can be canceled in the water vapor density result.

Single pulse modulation, 7-bit pulse modulation, and 15-bit pulse modulation are used for simulation. Specifications of the DIAL are given in Table 2. The HITRAN database of the water vapor absorption line is utilized for water vapor profile calculation. The aerosol profile is assumed as a U.S. standard model with $23 \mathrm{~km}$ ground visibility. The DIAL error is represented by total effective error including random error such as signal detection error, and systematic error such as temperature relative error and wavelength tuning error.

Table 2. The $\mathrm{H}_{2} \mathrm{O}$ DIAL simulation parameters

\begin{tabular}{|l|l|}
\hline Online wavelength & $829.054 \mathrm{~nm}$ \\
\hline Offline wavelength & $829.124 \mathrm{~nm}$ \\
\hline Pulse energy & $1 \mu \mathrm{J}$ \\
\hline Pulse width & $100 \mathrm{~ns}$ \\
\hline Repetition rate & $10 \mathrm{kHz}$ \\
\hline Accumulation time & $10 \mathrm{~min}$ \\
\hline Sky spectral radiance & $12.1 \mathrm{~mW} / \mathrm{m}^{2} / \mathrm{nm} / \mathrm{sr}$ \\
\hline Total opt. efficiency-Receiver & $29 \%($ day-time $)$ \\
\hline Quantum efficiency & $45 \%$ \\
\hline Telescope diameter & $35 \mathrm{~cm}$ \\
\hline Field of view & $224 \mu \mathrm{rad}$ \\
\hline
\end{tabular}

The total effective errors of three modulation models are plotted in Fig. 9. It is evident that the total effective of single pulse modulation is lowest in the low region. However, within $10 \%$ error, $7-$ bit pulse modulation, and 15-bit pulse modulation reach higher altitude compared to single pulse modulation. In detail, the maximum reached altitude within $10 \%$ error of single pulse modulation, 7-bit pulse modulation, and 15-bit pulse modulation are $1.5 \mathrm{~km}, 2.2 \mathrm{~km}$, and $2.8 \mathrm{~km}$, respectively. Though 7-bit pulse modulation is not optimum for lower/ higher region measurement compared to single/ 15-bit pulse modulation, it still maintains appropriate error through the whole region of the lower troposphere.

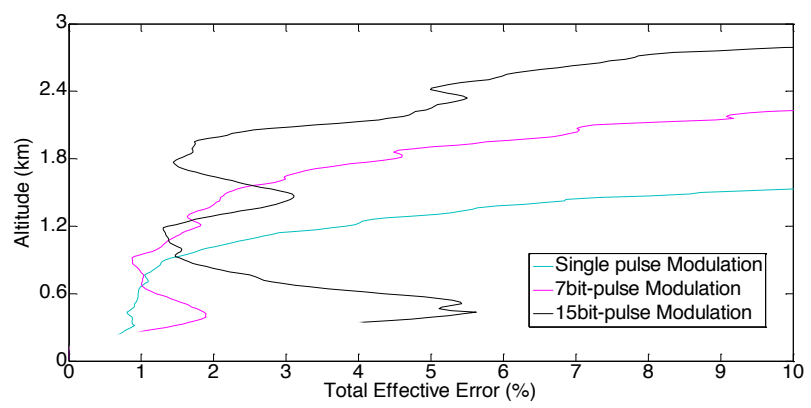

Figure 9. The total effective errors of single pulse modulation, 7-bit pulse modulation, and 15-bit pulse modulation.

\section{CONCLUSIONS}

In this research, we are developing a fielddeployable diode-laser-based water vapor DIAL. Photoacoustic absorption cell can be utilized for wavelength locking of DBR laser to online by spectroscopy experiment. The water vapor profile retrieved from observational data agrees with the referenced radiosonde data. In future, we will apply modulation pulse technique and wavelength locking system as well as improve the TA efficiency for the DIAL transmitter.

\section{ACKNOWLEDGEMENT}

This work was supported by JSPS KAKENHI Grant Number 26282115.

\section{REFERENCES}

[1] Repasky, K. S., Moen, D., Spuler, S., Nehrir, A. R., and Carlsten, J. L., 2013: Progress towards an Autonomous Field Deployable Diode-LaserBased Differential Absorption Lidar (DIAL) for Profiling Water Vapor in the Lower Troposphere, Remote Sens., 5(12), 6241-6259.

[2] Cochenour, B., Mullen, L., and Muth, J., 2011: Modulated pulse laser with pseudorandom coding capabilities for underwater ranging, detection, and imaging, Appl. Opt., 50(33), 6168-6178. 